\title{
Terahertz calculations for the Australian synchrotron
}

\author{
Chien Aun Chan ${ }^{\mathrm{a}}$, Samuel P. Mickan ${ }^{\mathrm{a}}$, Gywn Williams ${ }^{\mathrm{b}}$, Derek Abbott ${ }^{\mathrm{a}}$ \\ ${ }^{a}$ Centre for Biomedical Engineering (CBME) and Department of Electrical \& Electronic \\ Engineering, The University of Adelaide 5005, Australia.
}

${ }^{b}$ Free Electron Laser Facility, Jefferson Lab, 12000 Jefferson Avenue, Newport News, VA 23606, USA

\begin{abstract}
The development of terahertz radiation (T-rays) is spurring new applications in spectroscopy and imaging. To maximize the use of T-rays in more applications, a high average terahertz power is needed. Rather than using fast diodes or laser sources, this paper will show that a synchrotron can generate high average power T-rays. This is achieved by creating an electron bunch in the synchrotron ring with high intensity in the terahertz frequency region via Thomson scattering.
\end{abstract}

Keywords: Synchrotron, Terahertz, T-rays

\section{INTRODUCTION}

T-rays are a form of electromagnetic radiation, lying between millimeter waves and infrared radiation on the electromagnetic spectrum, as shown in the diagram below. T-ray radiation interacts in a unique way with matter, relating to rotational and vibration modes in gas, inter-domain movement in large biomolecules, and resonances in crystals. Trays have been used to identify water levels in living tissue, measure the real-time 'dressing' of electrons in semiconductors $^{1,2,3}$, detect malignant skin cancer ${ }^{4,5}$, and detect Anthrax-like spores in envelopes ${ }^{6}$. A newly accessible region of the electromagnetic spectrum will provide new technologies, especially if the low-power limitations of T-ray systems can be overcome.

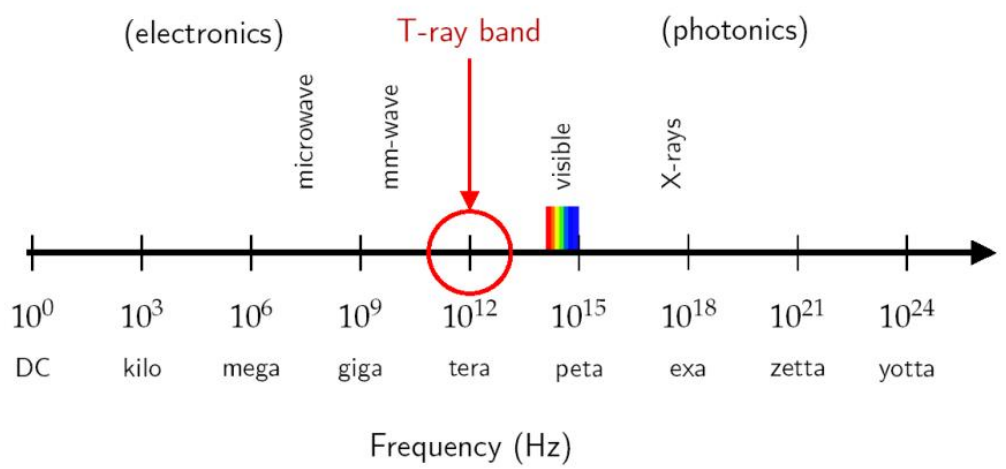

C. A. Chan Email: chien.chan@student.adelaide.edu.au; S. P. Mickan Email: spmickan@eleceng.adelaide.edu.au; G. Williams Email: gwyn@mailaps.org; D. Abbott Email: dabbott@eleceng.adelaide.edu.au 
In the past decade, broadband T-ray sources have been developed using fast diodes, femtosecond lasers and non-linear transmission lines, leading to increased interest in T-ray applications. However, the low average power generated by these methods ${ }^{7}$ (about $10^{-6} \mathrm{~W}$ ) is a major hurdle to be overcome. For example, the emerging technology of terahertz time domain spectroscopy (THz-TDS) provides the ability to identify a substance via its absorption spectra. With higher average power T-ray radiation, better detection of concealed weapons, hidden explosives and land mines can be performed.

In November 2002, the Jefferson Laboratory in the USA produced high average power broadband emission from subpicosecond electron bunches in an accelerator by using the facility's Energy Recovery Linac (ERL). The average power obtained was nearly 20 Watts, many times larger than other T-ray sources ${ }^{7}$. Jefferson Laboratory's ERL is a superconducting radio-frequency electron accelerator. The ERL is designed to recover spent by the electron bunches. In the ERL, short bunches of electrons, each with a pulse width of about 500 femtoseconds, were accelerated to energies of about $40 \mathrm{MeV}$ and then accelerated through a bend using a powerful magnetic field. The acceleration of the electron bunches through this bend caused T-ray radiation to be generated with a peak power ${ }^{7}$ of $106 \mathrm{~W}$ and a peak frequency ${ }^{7}$ of $0.6 \mathrm{THz}$.

This paper models T-ray generation in a synchrotron ring. In the linear accelerator (linac) system that Jefferson Laboratory used, each pass of the electron beam through the magnetic bend carried new electrons. However, in a synchrotron ring, the same electrons pass through the magnetic bend repeatedly without the replacing of new electrons.

In this paper, terahertz frequencies are theoretically obtained by narrowing the electron bunch via Thompson scattering. As scattering can be achieved with an ultrafast laser, gated detection via the standard pump-probe method ${ }^{3,8,9}$ can be used. The pump-probe approach has the added benefit of reducing jitter.

\section{SYNCHROTRON RADIATION}

A synchrotron ring emits a broadband spectrum of light by accelerating bunches of electrons at relativistic speeds in the ring. The intensity of the synchrotron radiation can be quantified by its brightness and power. Power can be represent by units of either Watts $/ \mathrm{cm}^{-1}$ or photons $/ \mathrm{sec} / 0.1 \%$ bandwidth. However, brightness or brilliance is defined as power divided by emittance. Therefore, the brightness would have units of either Watts $/ \mathrm{cm}^{-1} / \mathrm{mm}^{2} / \mathrm{sr}^{\circ}$ photons $/ \mathrm{sec} / 0.1 \%$ bandwidth $/ \mathrm{mm}^{2} \mathrm{sr}$.

\subsection{Power}

The power emitted from a synchrotron ring can be calculated by using the following formula:

$$
P(\lambda)=4.38 \cdot 10^{14} I \cdot \theta \cdot B W \cdot\left(\frac{\rho}{\lambda}\right)^{1 / 3} \quad \text { photons } / \mathrm{sec}
$$

where $I$ is the current in amperes, $\theta$ is the horizontal collection angle in radians, $B W$ is the bandwidth in $\%, \lambda$ is the wavelength in microns and $\rho$ is the radius of the ring and it has the same units as $\lambda$. Equation (1) shows the power of the synchrotron radiation in photons/sec, but it can be converted to Watts by dividing $P$ by $\left(5.04 \times 10^{18} \times \lambda\right)$. For a current $(I)$ of 200 milliampere and a $0.1 \%$ bandwidth $(B W)$, Equation $(1)$ can be simplified as:

$$
P(\lambda)=\frac{0.1 \lambda \cdot 2\left(f_{p a}(\lambda)+f_{p e}(\lambda)\right)}{5.04 \cdot 10^{18} \lambda} \quad \text { Watts } / \mathrm{cm}^{-1}
$$

where the $0.1 \lambda$ term results from the $0.1 \%$ bandwidth and therefore,

$$
P(\lambda)=3.9682 \cdot 10^{-20}\left(f_{p a}(\lambda)+f_{p e}(\lambda)\right) \mathrm{Watts} / \mathrm{cm}^{-1}
$$


The parameters $f_{p a}(\lambda)$ and $f_{p e}(\lambda)$ are the parallel and perpendicular polarized flux per milliradian vertically, which depend on the value of $\lambda$ in microns.

\subsection{Flux}

The formulae for calculating the values of $f_{p a}(\lambda)$ and $f_{p e}(\lambda)$ are ${ }^{10}$ :

$$
\begin{aligned}
& f_{p e}(\lambda)=1.3256 \cdot 10^{16} E^{2} \theta_{h}(\lambda) k_{1}(\lambda) \\
& f_{p a}(\lambda)=1.3256 \cdot 10^{16} E^{2} \theta_{h}(\lambda) k_{2}(\lambda) .
\end{aligned}
$$

Here, $E$ is the synchrotron beam energy in units of electron volts, while $\theta_{h}(\lambda), k_{l}(\lambda)$ and $k_{2}(\lambda)$ are the horizontal emission angles in radians and the intermediate variables, which depend on the values of $\lambda$, are given as:

$$
\begin{gathered}
k_{1}(\lambda)=2.5 \cdot 10^{-10} \lambda^{2} \gamma^{2} \theta_{h}(\lambda)^{2} \cdot\left(1+10^{-6} \gamma^{2} \theta_{h}(\lambda)^{2}\right) \cdot\left(\exp \left(\frac{-x_{1}(\lambda)}{2}\right)+G_{1}(\lambda)\right)^{2} \\
k_{2}(\lambda)=5 \cdot 10^{-4} \lambda^{2} \cdot\left[\left(1+10^{-3} \gamma \theta_{h}(\lambda)\right)^{2} \cdot\left(\exp \left(\frac{-x_{1}(\lambda)}{2}\right)+G_{2}(\lambda)\right)\right]^{2}
\end{gathered}
$$

where $\gamma$ is the electron relativistic to rest mass ratio, which is equal to $1957 \times E$ and $E$ is the synchrotron beam energy in $\mathrm{GeV}$. Also $x(\lambda)$ is the ratio of critical wavelength over wavelength $\left(\lambda_{c} / \lambda\right)$. By definition, the critical wavelength is the wavelength which bisects the power spectrum and it is defined as $(5.59 \times \rho)$ (meters) $/ E^{3}(\mathrm{GeV})$. Consequently, we can define $x(\lambda)$ as:

$$
x(\lambda)=\frac{5.59 \rho}{10^{4} E^{3} \lambda}
$$

\subsection{Bessel functions}

In Equations (6) and (7), $G_{l}(\lambda)$ and $G_{2}(\lambda)$ are Bessel functions and $x_{l}(\lambda)$ is also used in obtaining the required Bessel functions and is defined as ${ }^{10}$ :

$$
x_{1}(\lambda)=\frac{1}{2} x(\lambda)\left(1+\left(10^{-6} \lambda^{2} \theta^{2}\right)^{3 / 2}\right)
$$

Note that $G_{l}(\lambda)$ is the Bessel function of order $1 / 3$, for light polarized perpendicularly to the orbit plane. However, $G_{2}(\lambda)$ is the Bessel function of order $2 / 3$, for light, polarized parallel to the orbit plane. By definition, the Bessel function will be an expression of the form:

$$
G(x)=x \int_{x}^{\infty} K_{5 / 3}(\eta) d(\eta)
$$

By knowing that $x$ is the ratio of the critical wavelength over wavelength, the integral over the spherical fractional order Bessel function in Equation (10) can now be expressed using the Kostroun algorithm ${ }^{11,12}$, with fractional order, $k$ :

$$
G_{k}(x)=\frac{1}{2}\left(\frac{e^{-x}}{2}+\sum_{r=1}^{\infty} e^{-x \cdot \cosh (0.5 r)} \cdot \cosh (0.5 k r)\right) \text {. }
$$




\subsection{Brightness}

As discussed earlier, brightness is defined as the ratio of power by emittance $(P(\lambda) / \mathcal{E})$. By definition, emittance is a measurement of the synchrotron radiation intensity lost due to divergence of the electron beam and its non-zero crosssectional size. However, in practical terms, the brightness is the flux divided by the opening angle and the area of the source. As a consequence, low emittance will result in high brightness of synchrotron radiation. The emittance, $\varepsilon$ can be expressed by using the following equation:

$$
\varepsilon=10^{-12} \theta_{\mathrm{h}} \theta_{v} S_{h} S_{v} \mathrm{~mm}^{2} \mathrm{sr}
$$

The $\theta_{h}$ and $\theta_{v}$ terms are the horizontal and vertical emission angles in milliradians while $S_{h}$ and $S_{v}$ are the horizontal and vertical source sizes in microns as shown in Figure 1. The horizontal and vertical source sizes can be obtained by using the equations:

$$
\begin{aligned}
& S_{h}=\sqrt{{S_{h i}{ }^{2}+S_{h p}^{2}+S_{h d i f f}^{2}}^{2}} \\
& S_{v}=\sqrt{{S_{v i}{ }^{2}+S_{v p}^{2}+S_{v d i f f}^{2}}^{2}}
\end{aligned}
$$

where $S_{h i}$ and $S_{v i}$ are the intrinsic horizontal and vertical source sizes, $S_{h p}$ and $S_{v p}$ are the source sizes due to projection and $S_{\text {hdiff }}$ and $S_{v d i f f}$ are the source sizes due to diffraction.

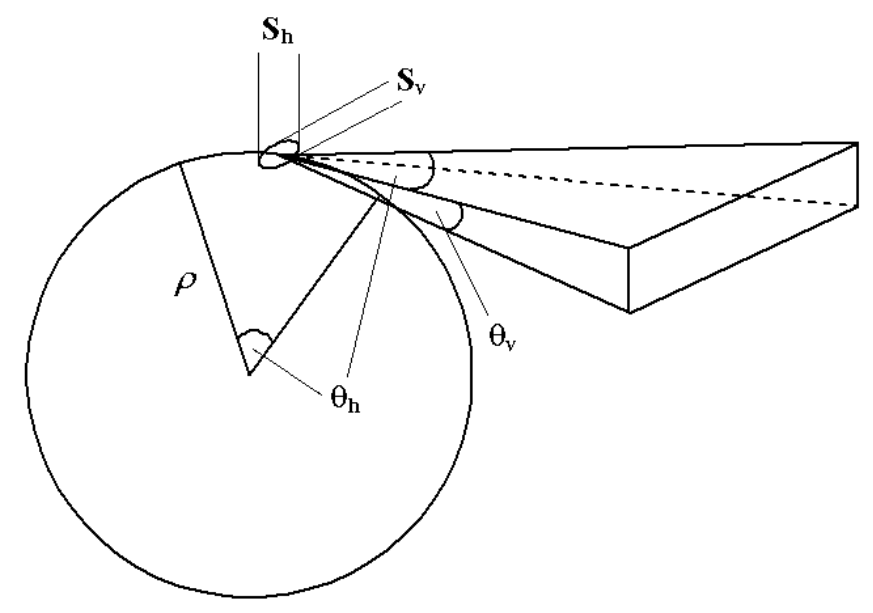

Figure 1. Synchrotron parameters. The circle in the diagram is the bending circle of a synchrotron ring with radius, $\rho$. The $S_{h}$ and $S_{v}$ are horizontal and vertical source sizes in microns and the $\theta_{h}$ and $\theta_{v}$ are the horizontal and vertical emission angles in milliradians.

To calculate the horizontal and vertical emission angle $\left(\theta_{h}\right.$ and $\left.\theta_{v}\right)$, the natural opening angle $\theta_{\text {nat }}$ and the beamline vertical and horizontal angle $\left(\theta_{h b}\right.$ and $\left.\theta_{v b}\right)$ are needed. Once these angles are determined, the horizontal emission angle will be the smallest angle of either $\theta_{n a t}$ or $\theta_{h b}$ and the vertical emission angle will be the smallest angle between $\theta_{n a t}$ and $\theta_{v b}$. In other words, the $\theta_{h}$ and $\theta_{v}$ must be less than or equal to $\theta_{n a t}$. The natural opening angle, $\theta_{n a t}$ can be determined by using Equation (14) where $\lambda$ is in microns and $\rho$ is in meters ${ }^{10}$,

$$
\theta_{\text {nat }}=1.66188 \cdot\left(\frac{10^{3} \lambda}{\rho}\right)^{1 / 3}
$$




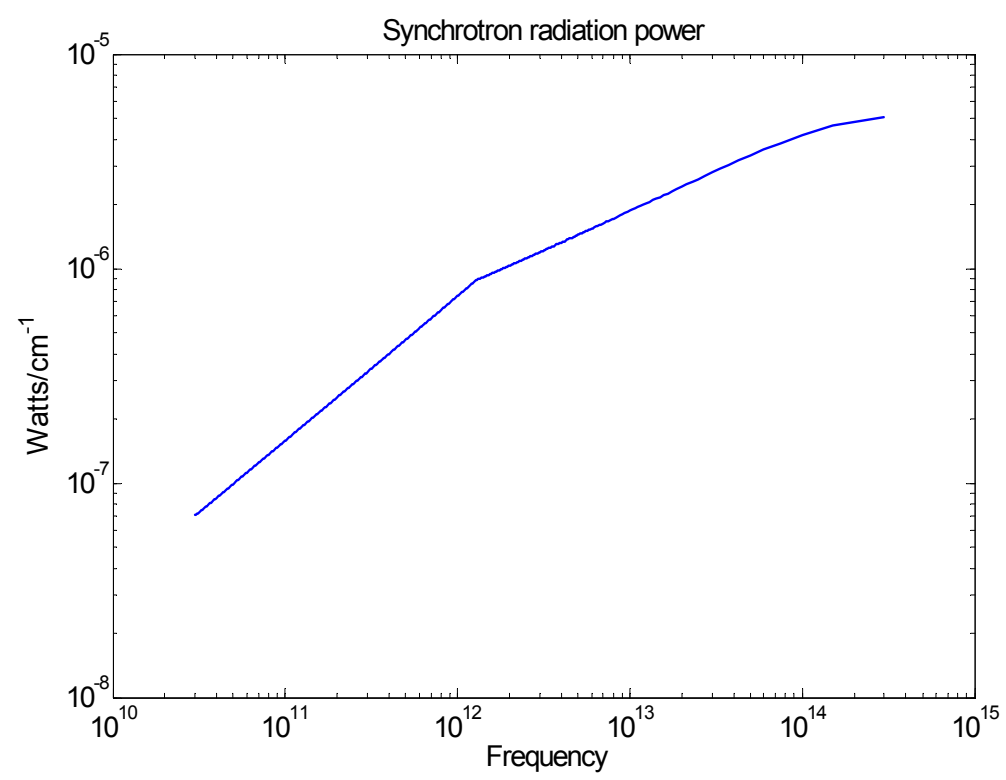

Figure 2. Synchrotron radiation power. For a current of 0.2 ampere, bandwidth of $0.1 \%$, sources size of 500 microns (horizontal) and 200 microns (vertical) and a 60 mradian (horizontal) by 60 mradian (vertical) collection angles, Equation (3) is used to generate the graph with Watts $/ \mathrm{cm}^{-1}$ versus frequency where $\mathrm{cm}^{-1}$ represents the wavenumber. Synchrotron radiation power is higher for increased spectral frequency. At a frequency of one terahertz $\left(10^{12} \mathrm{~Hz}\right)$, the intensity is around $10^{-6}$ Watts $/ \mathrm{cm}^{-1}$. This demonstrates that only weak T-ray power is generated in a regular synchrotron.

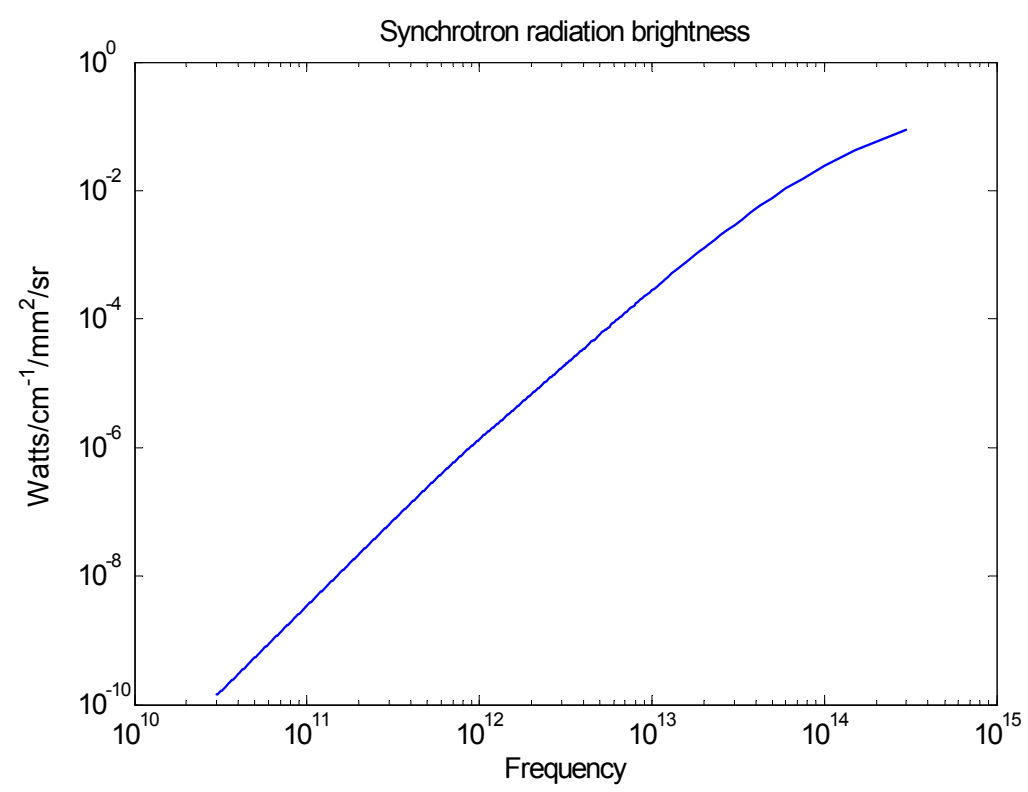

Figure 3. Synchrotron radiation brightness. By definition, the brightness equals to the power divided by emittance $(P / \mathcal{E})$. Therefore, with the same parameters used in Figure 2, the synchrotron brightness curve has the same characteristic as the synchrotron power curve. Similar to Figure 2, the synchrotron brightness intensity will increase when the frequency increases and this also shows a weak intensity occurs in the terahertz frequency range. 
Figure 2 and Figure 3 show the synchrotron radiation power and brightness plot against spectral frequency. Moreover, it can be clearly seen that the synchrotron radiation is weak in the terahertz $\left(10^{12} \mathrm{~Hz}\right)$ frequency region. To obtain high intensity synchrotron radiation, Thomson scattering is needed.

\section{THOMSON SCATTERING FOR T-RAY GENERATION}

The spectra of Figures 2 and 3 were derived from the theory of the passage of a single electron. In practice many electrons circulate together in bunches, adding another time-scale to the calculation. The squared Fourier transform of a Gaussian pulse will yield the intensity of the synchrotron radiation as shown in Figures 2 and 3. If the electron Gaussian pulse is sliced to have a shorter duration, the Fourier transform of the time domain characteristic will give a stronger intensity in terahertz region. If the electron bunch's dimensions are small compared to the wavelength of observation, the Fourier transform of the electron bunch will reach the terahertz region after slicing. The technique that is used to slice the electron bunch is known as locked laser Thomson scattering. A $500 \mathrm{MHz}$ mode-locked laser will shoot into a certain part of the synchrotron ring and slice the electron bunches to become narrower. When the size-reduced electron bunches are viewed in the frequency domain, an enhancement occurs in the frequency domain plot and the frequency reaches the terahertz region. The coherent enhancement factor, Nof Thomson scattering is given as:

$$
N=1-f(\omega)+f_{s} n_{e} f(\omega)
$$

where $n_{e}$ is the number of electrons per bunch, $f_{s}$ is the slicing factor. If the electron bunch is sliced by 200 femtoseconds every 20 picoseconds, the slicing factor $f_{s}$ will be 0.01 . Here, $f(\omega)$ represents the Fourier transform calculation, which is given by ${ }^{10}$ :

$$
f(\omega)=\exp \left(\frac{-4 \pi^{2} \sigma^{2}}{\lambda^{2}}\right)
$$

where $\lambda$ represents the wavelength in microns and $\sigma$ is the bunch sigma value in microns. The bunch $\sigma$ is given by:

$$
\sigma=\frac{0.3 L_{f w h m}}{2.2}
$$

where the $L_{f w h m}$ is the bunch length full width half maximum value in femtoseconds. When the coherent enhancement factor has been determined, the new power spectrum of the synchrotron radiation from Equation (3) is now:

$$
P^{\prime}(\lambda)=P(\lambda) \cdot N
$$

\section{MATLAB SIMULATION RESULT}

From Figure 4 and Figure 5, it can be seen that the synchrotron radiation power and brightness graphs occur to have an enhancement area. This enhancement area is not present before Thomson scattering as we can see by comparing Figures 4 and 5 with Figures 2 and 3. These enhancement areas occur due to the effect of narrowing the electron bunches via Thomson scattering. When the slices electron bunches become thinner and smaller, the power spectra of the electron bunch will result a high intensity in terahertz frequency region.

From Figure 4, a power of about $13.5 \mathrm{Watts} / \mathrm{cm}^{-1}$ is generated at $10^{12} \mathrm{~Hz}$. However, the peak in Figure 5 does not occur in the terahertz frequency region. A brightness of about 25 Watts $/ \mathrm{cm}^{-1} / \mathrm{mm}^{2} / \mathrm{sr}$ is generated but this is much higher than the existing one in Figure 3, which is around $1.5 \cdot 10^{-6} \mathrm{Watts} / \mathrm{cm}^{-1} / \mathrm{mm}^{2} / \mathrm{sr}$. As a consequence, this demonstrates that the Thomson scattering slicing method is an efficient way of producing T-ray power using synchrotron radiation. 


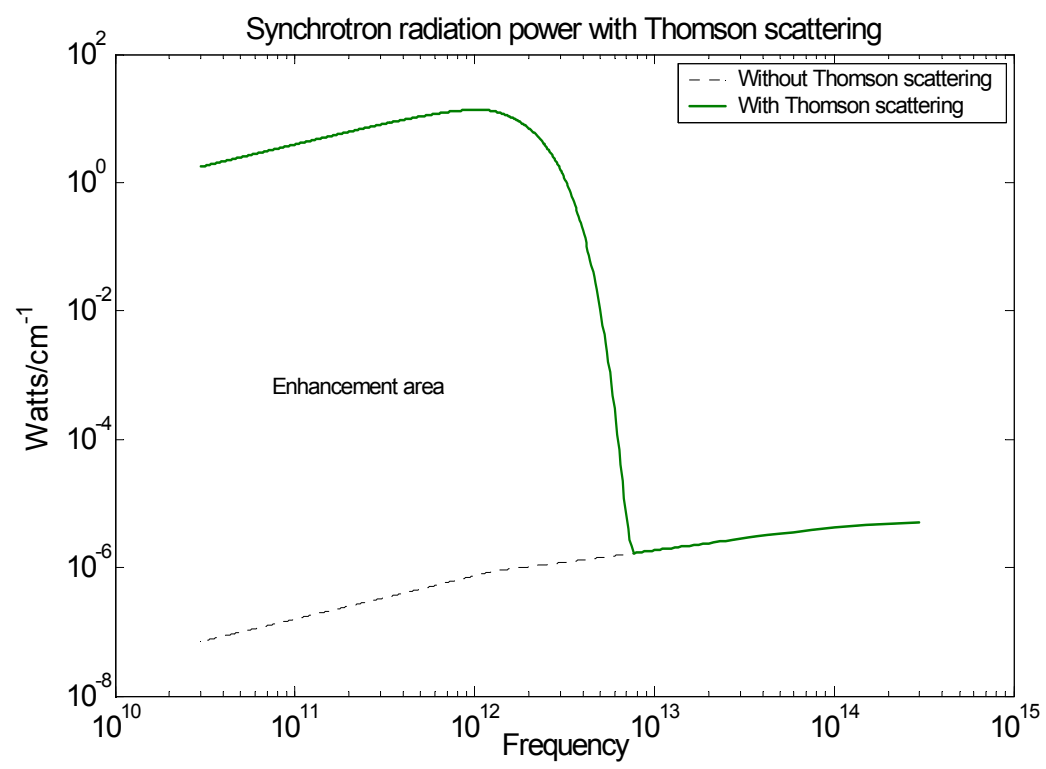

Figure 4. Synchrotron radiation power with Thomson scattering. Using Equation (18), with $f_{s}=0.01$, an enhancement area occurs in the graph. The graph with a solid line represents the synchrotron radiation power after Thomson scattering has been applied and the dotted line represents the synchrotron power before Thomson scattering. The graph also shows a peak occurs in the terahertz frequency region.

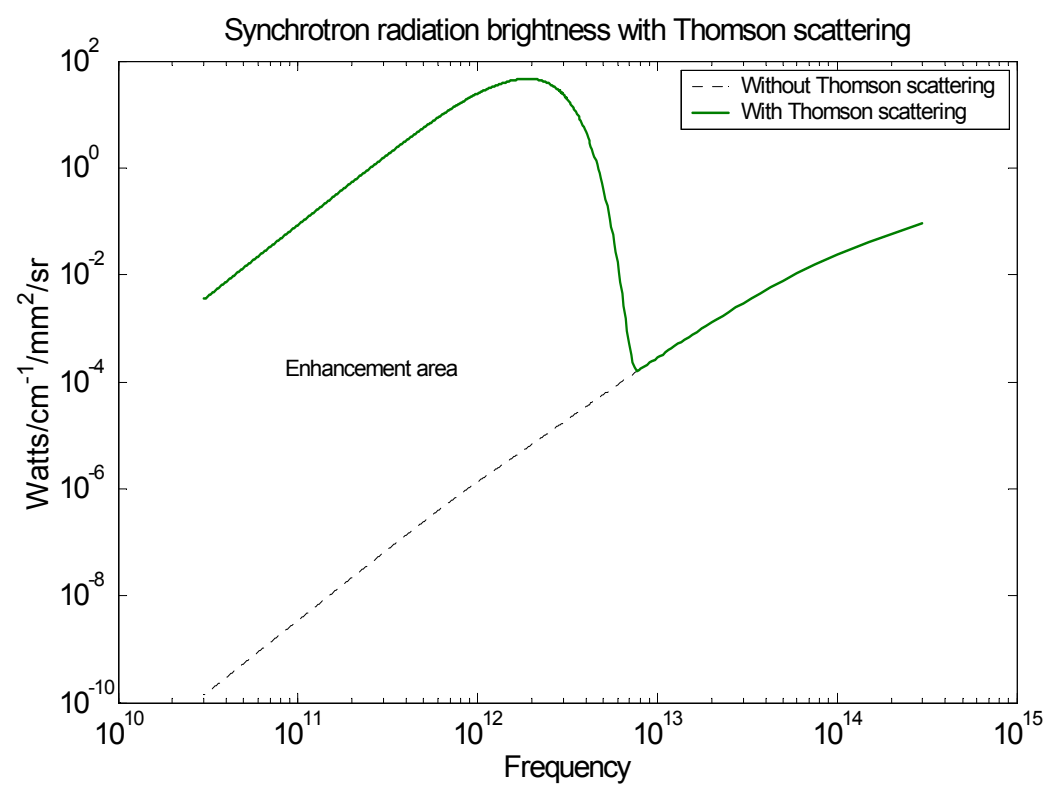

Figure 5. Synchrotron radiation brightness with and without Thomson Scattering. Using the same coherent enhancement factor, $N$ multiplied by the brightness in Figure 3, with high brightness intensity is generated as demonstrated in the above graph. The graph shows an enhancement similar to that in Figure 4 and is just a different way of representing the synchrotron radiation intensity. 


\section{CONCLUSION}

We have shown the calculations required for estimating T-ray power output from a synchrotron using Thomson scattering. Moreover, high synchrotron T-ray power will maximize the application of T-rays in several fields. Note that the calculated power $P(\lambda)$ depends on many parameters such as the bending radius, beamline vertical and horizontal angle, bunch length, source sizes, etc. In practice, many parts of the synchrotron ring need to be considered such as the bending magnet, booster ring, linear accelerator electron gun, and storage ring situation.

\section{ACKNOWLEDGEMENTS}

The support of The University of Adelaide and the Australian Research Council are gratefully acknowledged.

\section{REFERENCES}

1. D. Grischkowsky, S. Keiding, M. V. Exter, C. Fattinger, "Far-infrared time-domain spectroscopy with terahertz beams of dielectrics and semiconductors," Journal of the Optical Society of America B: Optical Physics 7(10), pp. 2006-2015, 1990.

2. D. M. Mittleman, J. Cunningham, M. C. Nuss, M. Geva, "Noncontact semiconductor wafer characterization with the terahertz Hall effect," Applied Physics Letters 71(1), pp. 16-18, 1997.

3. S. P. Mickan, D. Abbott, J. Munch, X. C. Zhang, and T. van Doorn, "Analysis of system trade-offs for terahertz imaging," Microelectronics Journal (Elsevier) 31(7), pp. 503-514,2000.

4. R. M. Woodward, B. E. Cole, V. P. Walace, D. D. Arnone, R. J. Pye, E. H. Linfield, M. Pepper, "Terahertz pulse imaging of in reflection geometry of human skin cancer and skin tissue," Physics in Medicine and Biology 47, pp. 3853-3863, 2002.

5. B. Ferguson, S. Wang, D. Gray, D. Abbott, X.-C. Zhang, "Identification of biological tissue using chirped probe THz imaging," Microelectronics Journal (Elsevier) 33, pp. 1043-1051, 2002.

6. S. Wang, B. Ferguson, C. Mannella, D. Gray, D. Abbott, X.-C. Zhang, "Powder detection using THz imaging," OSA Trends in Optics and Photonics (TOPS) 73, pp. 132, 2002.

7. G. L. Carr, Michael C. Martin, Wayne R. McKinney, K. Jordan, George R. Neil, and G. P. Williams, "High-power terahertz radiation from relativistic electrons," Nature 420, pp.153-156, 2002.

8. S. P. Mickan, K. S. Lee, T. M. Lu, J. Munch, D. Abbott, and X.-C. Zhang, "Double modulated differential THzTDS for thin film dielectric characterization," Microelectronics Journal (Elsevier) 33(12), pp. 1033-1042, 2002.

9. S. P. Mickan, A. Menikh, H. Liu, C. A. Mannella, D. Abbott, J. Munch, X.-C. Zhang, "Label-free bioaffinity detection using terahertz technology," Physics in Medicine and Biology 47(21), pp. 3789-3795, 2002.

10. G. P. Williams, "FAR-IR/THz radiation from the Jefferson Laboratory, energy recovered linac, free electron laser," Review of Scientific Instruments 73(3), pp. 1461-1463, 2002.

11. V. Kostroun, "Simple numerical evaluation of modified Bessel functions $\mathrm{K}_{\mathrm{v}}(\mathrm{x})$ of fractional order and the integral $\mathrm{x}^{\infty} \mathrm{K}_{\mathrm{v}}(\eta) \mathrm{d}(\eta), "$ Nuclear Instruments \& Methods (Elsevier) 172, pp. 371-374, 1980.

12. H. N. Rutt, "On the evaluation of modified Bessel functions of the second kind and fractional order for synchrotron radiation calculations," Nuclear Instruments \& Methods in Physics Research Section A (Elsevier), 2003 (In Press). 


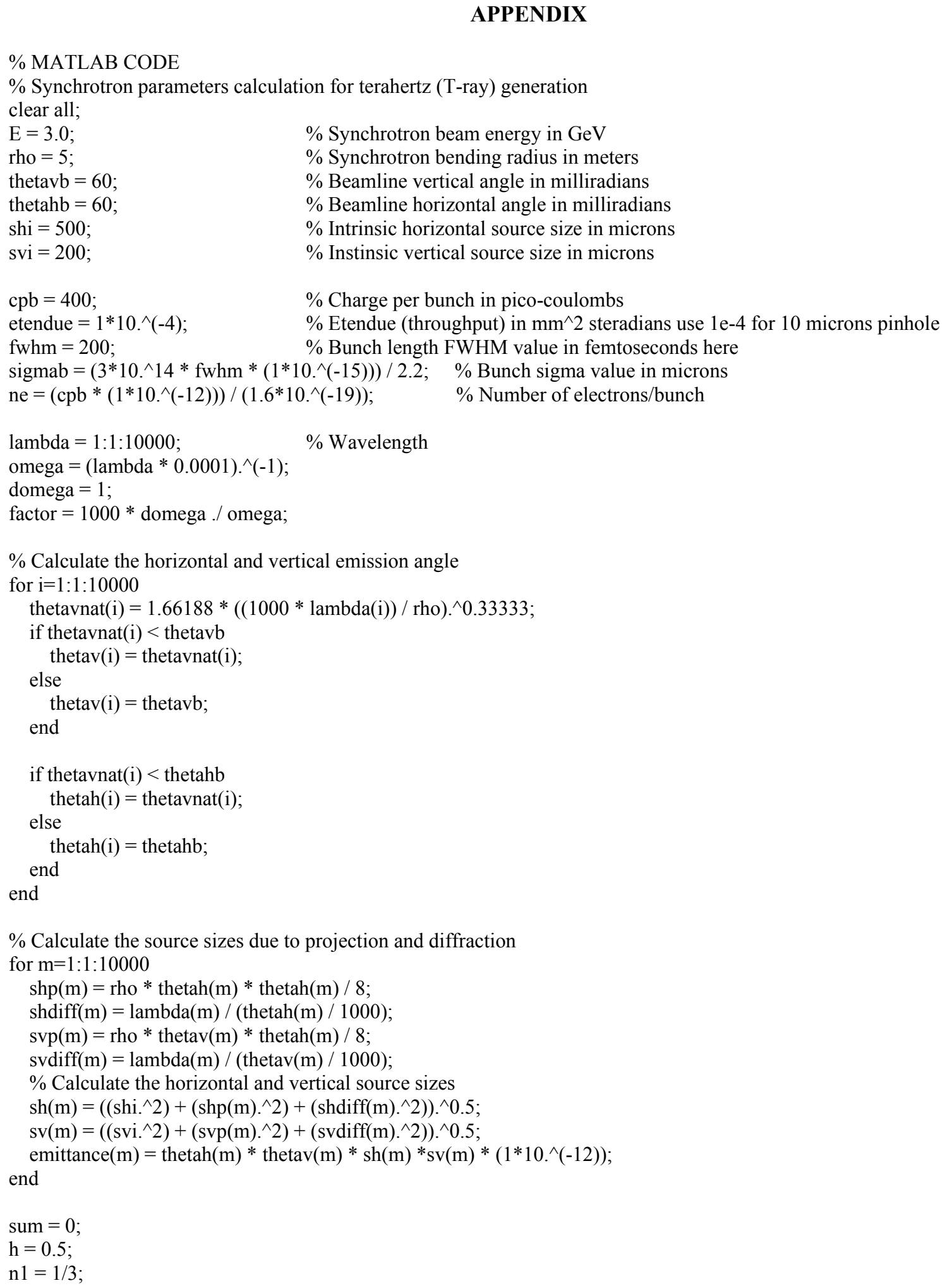




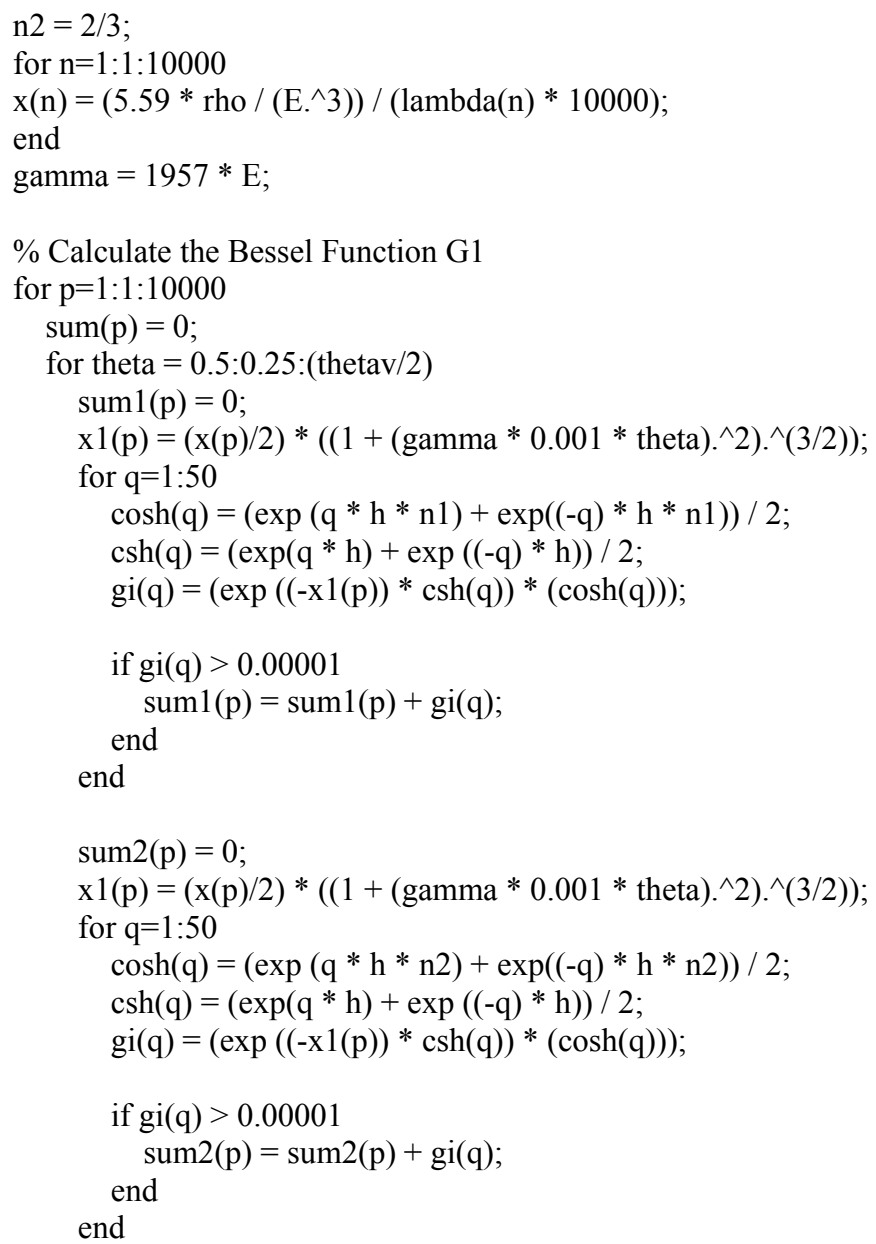

$\%$ Calculate the flux polarized parallel (fpa) or perpendicular (fpe) per milliradians vertically, in other words from $\%$ (theta- 0.5$)<$ theta $<$ (theta +0.5$)$ in milliradians. To do this, we multiply $\mathrm{k} 1$ and $\mathrm{k} 2$ by 0.001 radians

$\mathrm{k} 1(\mathrm{p})=0.001 * \mathrm{x}(\mathrm{p}) .^{\wedge} 2 *(($ gamma $* 0.001 *$ theta $) . \wedge 2) *(1+($ gamma $* 0.001 *$ theta $) . \wedge 2) *((\mathrm{~h} *((\exp (-(\mathrm{x} 1(\mathrm{p}))) /$ 2) $+\operatorname{sum} 1(\mathrm{p}))) \cdot \wedge 2)$

$\mathrm{k} 2(\mathrm{p})=0.001 * \mathrm{x}(\mathrm{p}))^{\wedge} 2 *((1+($ gamma $* 0.001 *$ theta $) . \wedge 2) * \mathrm{~h} *((\exp (-(\mathrm{x} 1(\mathrm{p}))) / 2)+\operatorname{sum} 2(\mathrm{p}))) .{ }^{\wedge} 2$;

$\mathrm{fpe}(\mathrm{p})=\left(1.3256 * 10 .^{\wedge} 16\right) * \mathrm{E} .^{\wedge} 2 *$ thetah $(\mathrm{p}) * \mathrm{k} 1(\mathrm{p})$;

$\mathrm{fpa}(\mathrm{p})=\left(1.3256 * 10 .^{\wedge} 16\right) * \mathrm{E} . \wedge 2 * \operatorname{thetah}(\mathrm{p}) * \mathrm{k} 2(\mathrm{p})$;

$\operatorname{sum}(p)=\operatorname{sum}(p)+2 *(f p a(p)+f p e(p)) ;$

end

end

$\%$ sum gives the result in photons/sec into $0.1 \%$ bandwidth using the smaller

$\%$ of the natural and actual vertical opening angles, and the smaller of the

$\%$ natural and actual horizontal angles, but the latter can be changed

$\%$ if we need Watts per $\mathrm{cm}-1$, then multiply by the bw factor and convert

for $1=1: 1: 10000$

$\operatorname{watts}(1)=$ factor $(1) * \operatorname{sum}(1) /((5.04 * 10 . \wedge 24) * \operatorname{lambda}(1) * 0.000001)$;

$\%$ we have total flux, now calculate the brightness

bright(1) = sum(l) / emittance $(1)$; 


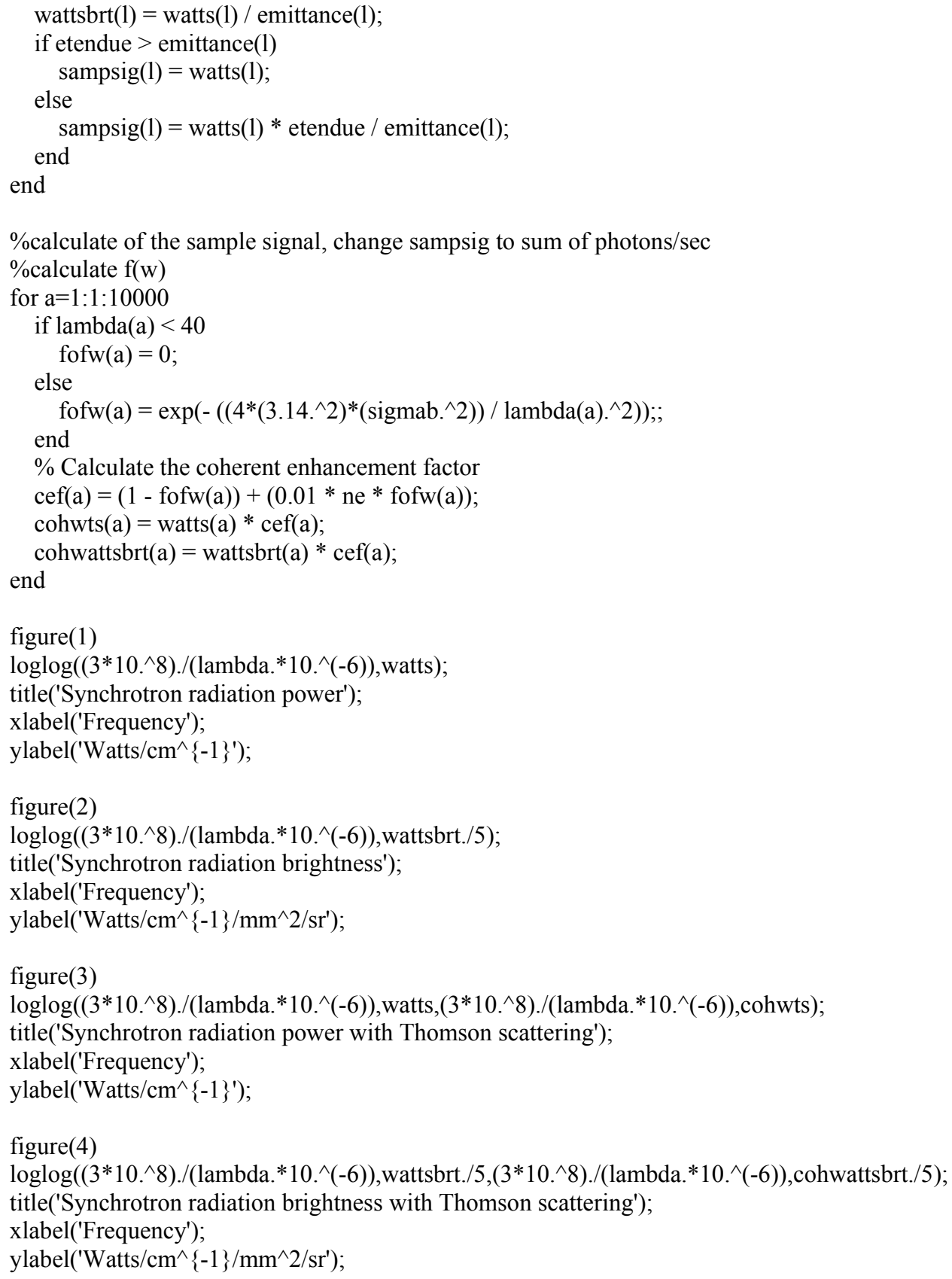

